\title{
Whole-Genome Sequencing of Shiga Toxin-Producing Escherichia coli OX18 from a Fatal Hemolytic Uremic Syndrome Case
}

\author{
Kenichi Lee, Atsushi Iguchi, Kazuhiro Uda, Sohshi Matsumura, Isao Miyairi, \\ Kenji Ishikura, Makoto Ohnishi, Junji Seto, Kanako Ishikawa, Noriko Konishi, \\ Hiromi Obata, Ichiro Furukawa, Hiromi Nagaoka, Hirotaka Morinushi, Natsuki Hama, \\ Ryohei Nomoto, Hiroshi Nakajima, Hideaki Kariya, Mitsuhiro Hamasaki, Sunao lyoda
}

We report a fatal case of hemolytic uremic syndrome with urinary tract infection in Japan caused by Shiga toxinproducing Escherichia coli. We genotypically identified the isolate as $\mathrm{OX} 18: \mathrm{H} 2$. Whole-genome sequencing revealed 3 potentially pathogenic lineages (OX18:H2, H19, and $\mathrm{H} 34$ ) that have been continuously isolated in Japan.

Shiga toxin-producing Escherichia coli (STEC) is a Consequential foodborne pathogen worldwide. The most prevalent STEC O serogroups-O157, O26, O111, O103, O121, O145, and O45-cause severe symptoms, including bloody diarrhea and hemolytic uremic syndrome (HUS). These STECs usually carry the locus of enterocyte effacement (LEE) region, which is required for intimate bacterial adherence to host epithelial cells (1). However,

Author affiliations: National Institute of Infectious Diseases,

Tokyo, Japan (K. Lee, M. Ohnishi, S. Iyoda); University of

Miyazaki, Miyazaki, Japan (A. Iguchi); National Center for Child

Health and Development, Tokyo (K. Uda, S. Matsumura, I. Miyairi,

K. Ishikura); Tokyo Metropolitan Children's Medical Center, Tokyo

(K. Uda); Kanagawa Children's Medical Center, Kanagawa, Japan

(S. Matsumura); Kitasato University School of Medicine, Tokyo

(K. Ishikura); Yamagata Prefectural Institute of Public Health,

Yamagata, Japan (J. Seto); Ibaraki Prefectural Institute of Public

Health, Ibaraki, Japan (K. Ishikawa); Tokyo Metropolitan Institute

of Public Health, Tokyo (N. Konishi, H. Obata); Kanagawa

Prefectural Institute of Public Health, Kanagawa (I. Furukawa);

Shizuoka Institute of Environment and Hygiene, Shizuoka, Japan

(H. Nagaoka, H. Morinushi); Kobe Institute of Health, Hyogo,

Japan (N. Hama, R. Nomoto); Okayama Prefectural Institute for

Environmental Science and Public Health, Okayama, Japan

(H. Nakajima, H. Kariya); Fukuoka Institute of Health and

Environmental Sciences, Fukuoka, Japan (M. Hamasaki)
LEE-negative STEC serotypes, including O104:H4 and O113:H21, can also cause outbreaks or severe cases $(2,3)$. Although most severe cases develop from intestinal tract infections, HUS cases related to urinary tract infections have been reported (4). We report a fatal case of HUS in Japan caused by a LEEnegative strain identified as OX18:H2.

\section{The Case}

In 2017, an 8-year-old girl in Japan was hospitalized for a urinary tract $E$. coli infection, which was treated with ceftazidime. Two days after hospitalization, she became unconscious. Laboratory results revealed anemia (hemoglobin $10.5 \mathrm{~g} / \mathrm{dL}$ ) with schistocytes; low platelet count $\left(3.8 \times 10^{4} / \mu \mathrm{L}\right)$; and elevated creatinine $(1.38 \mathrm{mg} / \mathrm{dL})$, total bilirubin $(1.7 \mathrm{mg} / \mathrm{dL})$, and lactate dehydrogenase $(1,848 \mathrm{U} / \mathrm{L})$. Magnetic resonance imaging of her head showed hyperintensity in the basal ganglia and thalamus, suggesting edema and necrosis. From the urine sample, we isolated a LEE-negative STEC (strain JNE170426) carrying the Shiga toxin 2 gene (stx2). On the basis of these findings we diagnosed her condition as HUS with urinary tract infection. We performed intravenous high-dose methylprednisolone therapy, plasma exchange, and hemodialysis for HUS encephalopathy and renal failure, but after 12 days of intensive therapy, she died of HUS encephalopathy.

The isolated STEC did not show agglutination against commercial O1-O188 antisera (Denka Company Ltd., https://www.denka.co.jp; Statens Serum Institut, https://en.ssi.dk). However, comprehensive PCR-based O serogrouping (5) revealed that the isolate was classified into OX18, an atypical $O$ serogroup originally identified from a nonpathogenic E. coli strain from 
a healthy sow (6). Using OX18-specific PCR screening of O-untypeable STEC isolates obtained during 2007-2019 by the National Institute of Infectious Diseases in Japan, we found 25 additional STEC OX18 isolates (Table). To characterize these isolates, we performed whole-genome sequencing (WGS) using MiSeq (Illumina, https:// www.illumina.com). WGS results were analyzed as described elsewhere $(7,8)$ with slight modification. We used BactSNP version 1.0.2 (http:/ / platanus.bio.titech. ac.jp/bactsnp) (9) and Gubbins version 2.4 .1 (https:// sanger-pathogens.github.io) (10) for core genome SNP extraction. Public database strains used for the phylogenetic analysis are shown in Appendix Table 1 (https: / wwwnc.cdc.gov/EID/article/27/5/20-4162-App1. pdf). We deposited draft genome sequences and shortread sequencing data into the DDBJ/National Center for Biotechnology Information/European Nucleotide Archive database (BioProject accession no. PRJDB10421; Sequence Read Archive accession no. DRA010812).

In silico analysis revealed that none of the STEC OX18 isolates carried LEE; we classified them into 5 H-genotypes: H2 $(\mathrm{n}=2)$, H8 $(\mathrm{n}=1), \mathrm{H} 19(\mathrm{n}=20)$, H28 ( $n=1)$, and H34 ( $=2)$ (Table). Core-genome SNP phylogeny revealed that OX18 isolates with the same H-types formed closely related groups (Table; Figure). Isolates from patients belonged to OX18:H2, $\mathrm{H} 19$, and $\mathrm{H} 34$; isolates belonging to OX18:H8 and $\mathrm{H} 28$ were obtained from asymptomatic carriers. The
OX18 isolate from the case-patient who died of HUS (strain JNE170426) belonged to $\mathrm{H} 2$ and carried stx2a and several virulence genes, including STEC autoagglutination adhesin (saa), subtilase toxin (sub), enterohemolysin $(e h x)$, and serine protease (espP) (Appendix Table 2). These regions showed high similarity $(>99 \%)$ to a large plasmid of STEC O104:H21 strain CFSAN002236 (11). Therefore, these virulence factors are likely to be encoded in similar plasmids.

On the other hand, the other OX18:H2 isolate (strain JNE133347) from an asymptomatic carrier did not carry the virulence genes described above but carried genes for Shiga toxin 2e (stx2e), heat-stable enterotoxin (st), and Pap fimbriae (pap). Of note, the other isolates obtained from HUS belonged to OX18:H19 and were phylogenetically close to OX18:H2 (Figure). The OX18:H19 lineage showed a similar virulence profile to the OX18:H2 isolate from the fatal HUS case, and carried saa, sub, ehx, and espP virulence genes on plasmid-like elements. All bovine isolates in our study were grouped into this serotype. OX18:H19 isolates from humans and bovines could not be distinguished by their lineages, suggesting that cattle can be a reservoir for that lineage. We identified OX18:H34 in isolates that carried pap as an adhesin from a patient with bloody diarrhea and from swine. The other isolates, from asymptomatic carriers, we classified into $\mathrm{H} 8$ and H28. The OX18:H8 isolate carried saa, sub, ehx, and espP

Table. OX18 isolates used in study of whole-genome sequencing of Shiga toxin-producing Escherichia coli OX18 from a fatal hemolytic uremic syndrome case, Japan*

\begin{tabular}{|c|c|c|c|c|c|c|c|c|c|c|}
\hline \multirow[b]{2}{*}{ Strain } & \multirow{2}{*}{$\begin{array}{c}\text { Year } \\
\text { isolated }\end{array}$} & \multirow[b]{2}{*}{ Source } & \multirow[b]{2}{*}{ Symptoms } & \multirow{2}{*}{$\begin{array}{c}\mathrm{H} \\
\text { genotype }\end{array}$} & \multirow{2}{*}{$\begin{array}{l}\text { Phylogenetic } \\
\text { group }\end{array}$} & \multirow[b]{2}{*}{ MLST } & \multicolumn{2}{|c|}{ stx subtype } & \multicolumn{2}{|c|}{ Accession no. } \\
\hline & & & & & & & $s t \times 1$ & $s t x 2$ & Draft genome & Short reads \\
\hline JNE101081 & 2010 & Human & BD & H34 & $E$ & 9185 & $1 a$ & ND & BNCS00000000 & SAMD00244533 \\
\hline JNE130471 & NA & Swine & NA & $\mathrm{H} 34$ & $E$ & 9185 & $1 a$ & ND & BNCT00000000 & SAMD00244534 \\
\hline JNE130573 & 2012 & Human & D & $\mathrm{H} 19$ & B1 & 205 & ND & $2 a$ & BNCU00000000 & SAMD00244535 \\
\hline JNE133347 & 2012 & Human & $A C$ & $\mathrm{H} 2$ & B1 & 9397 & ND & $2 \mathrm{e}$ & BNCV00000000 & SAMD00244536 \\
\hline JNE150598 & 2015 & Human & BD & $\mathrm{H} 19$ & B1 & 205 & ND & $2 a$ & BNCW00000000 & SAMD00244537 \\
\hline JNE151350 & 2015 & Human & $\mathrm{AC}$ & $\mathrm{H} 19$ & B1 & 205 & ND & $2 d$ & BNCX00000000 & SAMD00244538 \\
\hline JNE170426 & 2017 & Human & HUS, death & $\mathrm{H} 2$ & B1 & 847 & ND & $2 a$ & BNCY00000000 & SAMD00244539 \\
\hline JNE180342 & 2018 & Human & $\mathrm{AC}$ & $\mathrm{H} 8$ & B1 & Novel & $1 \mathrm{a}$ & $2 d$ & BNCZ00000000 & SAMD00244540 \\
\hline JNE181771 & 2018 & Human & HUS & $\mathrm{H} 19$ & B1 & 205 & ND & $2 a$ & BNDA00000000 & SAMD00244541 \\
\hline JNE182474 & 2018 & Human & BD & $\mathrm{H} 19$ & B1 & 205 & ND & $2 a$ & BNDB00000000 & SAMD00244542 \\
\hline JNE182523 & NA & Human & NA & $\mathrm{H} 19$ & B1 & 205 & ND & $2 a$ & BNDC00000000 & SAMD00244543 \\
\hline JNE191031 & 2019 & Human & BD & H19 & B1 & 205 & ND & $2 a$ & BNDD00000000 & SAMD00244544 \\
\hline JNE192124 & 2019 & Human & $A C$ & H19 & B1 & 205 & ND & $2 a$ & BNDE00000000 & SAMD0 \\
\hline JNE192333 & 2019 & Human & $A C$ & $\mathrm{H} 28$ & B1 & 1056 & $1 d$ & ND & BNDF00000000 & SAMDC \\
\hline A140161 & 2010 & Cattle & NA & H19 & B1 & 205 & ND & $2 a$ & BNDG00000000 & SAMD0 \\
\hline A140164 & 2010 & Cattle & NA & H19 & B1 & 205 & ND & $2 a$ & BNDH00000000 & SAMD00244548 \\
\hline A140165 & 2010 & Cattle & NA & H19 & B1 & 205 & ND & $2 a$ & BNDI00000000 & SAMD00244549 \\
\hline A140286 & 2012 & Cattle & NA & H19 & B1 & 205 & ND & $2 a$ & BNDJ00000000 & SAMD00244550 \\
\hline A140453 & 2010 & Cattle & NA & H19 & B1 & Novel & $1 a$ & $2 a$ & BNDK00000000 & SAMD00244551 \\
\hline A140462 & 2010 & Cattle & NA & H19 & B1 & 205 & $1 a$ & ND & BNDL00000000 & SAMD00244552 \\
\hline A140486 & 2014 & Cattle & NA & $\mathrm{H} 19$ & B1 & 205 & ND & $2 a \times 2 \dagger$ & BNDM00000000 & SAMD00244553 \\
\hline A150011 & 2014 & Cattle & NA & $\mathrm{H} 19$ & B1 & 205 & ND & $2 a \times 2 \dagger$ & BNDN00000000 & SAMD00244554 \\
\hline A150026 & 2014 & Cattle & NA & $\mathrm{H} 19$ & B1 & 205 & ND & $2 a \times 2 \dagger$ & BNDO00000000 & SAMD00244555 \\
\hline A150037 & 2015 & Cattle & NA & $\mathrm{H} 19$ & B1 & 205 & ND & $2 a \times 2 \dagger$ & BNDP00000000 & SAMD00244556 \\
\hline A150038 & 2015 & Cattle & NA & $\mathrm{H} 19$ & B1 & 205 & ND & $2 a \times 2 \dagger$ & BNDQ00000000 & SAMD00244557 \\
\hline A150039 & 2015 & Cattle & NA & $\mathrm{H} 19$ & B1 & 205 & ND & $2 a \times 2 \dagger$ & BNDR00000000 & SAMD00244558 \\
\hline
\end{tabular}

${ }^{*} \mathrm{AC}$, asymptomatic carrier; BD, bloody diarrhea; D, diarrhea; HUS, hemolytic uremic syndrome; NA, not available; ND, not detected.

$\dagger 2 a \times 2$, two copies of stx2a were detected. 


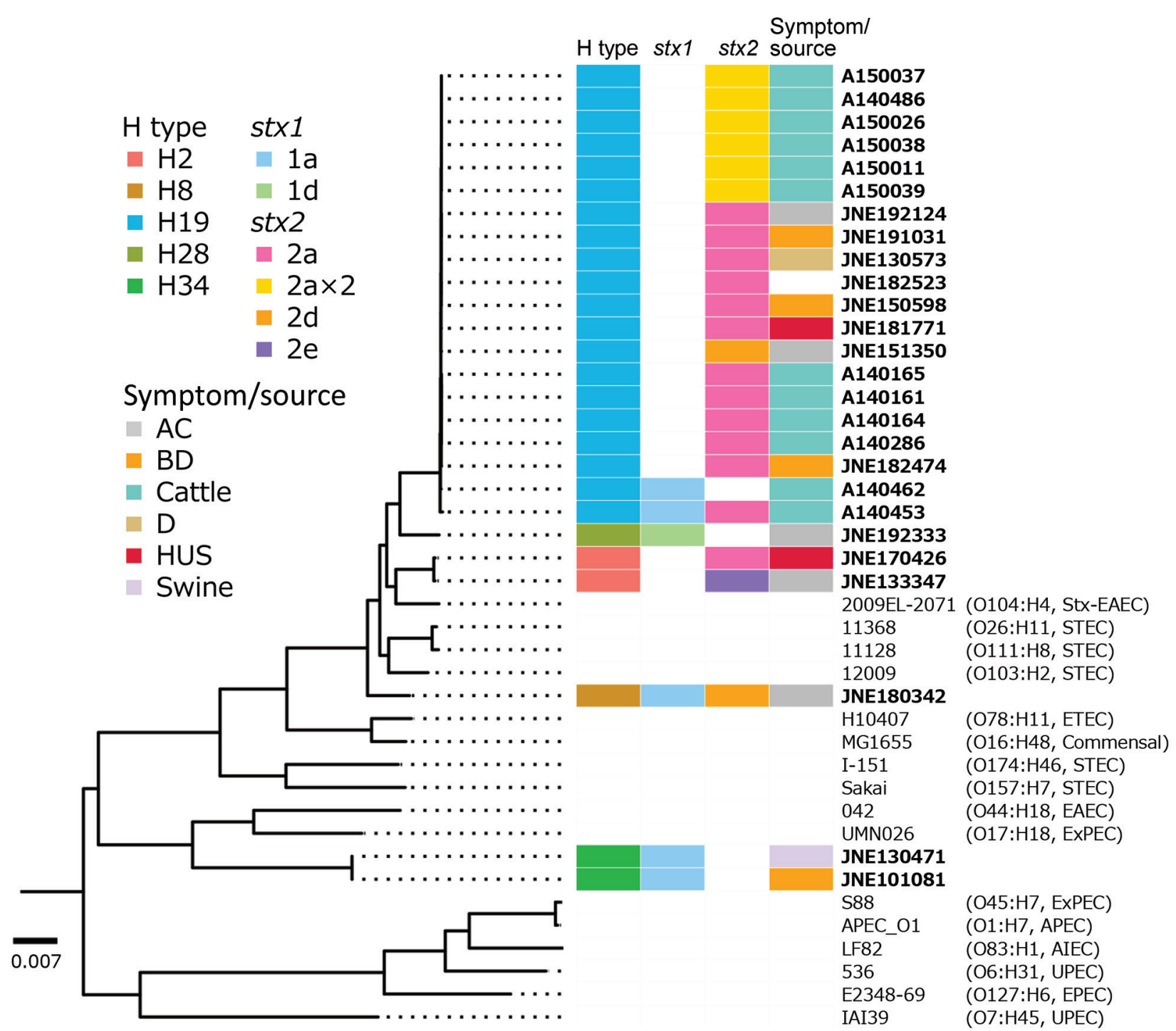

Figure. Maximum-likelihood phylogeny of STEC OX18 from a patient in Japan and other Escherichia coli strains. Isolate identifications of STEC OX18 are shown in bold. Colored boxes indicate collection countries, stx profiles, and symptoms of human carrier or source of the STEC OX18 isolates, as shown in the keys. Serotype and pathotype information of non-OX18 E. coli strains are shown in parentheses. The tree was rooted by E. fergusonii ATCC35469. AC, asymptomatic carrier; BD, bloody diarrhea; D, diarrhea; HUS, hemolytic uremic syndrome; STEC, Shiga toxin-producing E. coli; Stx, Shiga toxin. APEC, avian pathogenic E. coli; AIEC, adherent/ invasive E. coli; EAEC, enteroaggregative E. coli; EPEC, enteropathogenic E.coli; ExPEC, extraintestinal pathogenic E. coli; UPEC, uropathogenic $E$. coli. Scale bar indicates number of substitutions per site.

on plasmid-like elements, similar to the H19 lineage. Meanwhile, the OX18:H28 isolate did not carry adherence factors known in pathogenic E. coli, including LEE genes, saa, pap, aggR, afaD, F4, F6, F17, F18, or F41.

Among LEE-negative STEC isolates, saa-positive STEC has often been reported in patients with severe symptoms $(2,3)$. WGS analyses of saa-positive STEC O104:H21 and O113:H21 revealed that they carry a large plasmid $(>100 \mathrm{~kb})$ with several virulence genes, including saa and sub. Because the draft genomes of saa-positive OX18:H2 and H19 showed high similarity to the plasmid, it is plausible that they carry a similar large plasmid. The source or natural reservoir of these lineages was unclear. However, some OX18:H19 isolates have been obtained from cattle, suggesting that cattle or fecally contaminated foods can be a source of the infection. In addition to these lineages, OX18:H34 was found to cause severe symptoms in humans. We were unable to elucidate the pathogenesis and natural reservoir of the lineage because of the small sample size of our study; further studies are required. 


\section{Conclusion}

In this study, we report a HUS case with urinary tract infection caused by a STEC belonging to the emerging O serogroup OX18. Our retrospective survey revealed that the novel pathogenic STECs OX18:H2, H19, and H34 have been continually isolated from humans and cattle. However, commercial antisera cannot identify these lineages. Elucidating the transmission routes and natural reservoirs of the bacteria is essential to control infection. DNA-based serotyping methods, including $\mathrm{Og} / \mathrm{Hg}$ typing $(6,12,13)$ and whole-genome sequencing $(7,14,15)$, would be helpful for identification and surveillance of these potentially pathogenic lineages.

\section{Acknowledgments}

We are grateful to Saomi Ozawa, Yukie Nakajima, Ayumi Takemoto, and Yu Takizawa for technical assistance and WGS analyses. We are also grateful to Mai Sato, Koichi Kamei, Masaya Kubota, Naho Nishimura, and Satoshi Nakagawa for supporting clinical practice.

STEC isolates were kindly provided by the Department of Microbiology at the Miyagi Prefectural Institute of Public Health and Environment, the Gunma Prefectural Institute of Public Health and Environmental Sciences, the Kagawa Prefectural Research Institute for Environmental Sciences and Public Health, and the Fukuoka City Institute of Health and Environment.

This work was partially supported by the Research Program on Emerging and Re-emerging Infectious Diseases from the Japan Agency for Medical Research and Development (AMED) (grant number: JP20fk0108065), JSPS KAKENHI (18K15156 and 20K07506), and grants-inaid from the Ministry of Health, Labour, and Welfare of Japan (JPMH20HA1009).

\section{About the author}

Dr. Lee is a senior researcher in the Department of Bacteriology I, National Institute of Infectious Diseases in Tokyo. His primary research interests are the genomics and pathogenesis of Shiga toxin-producing Escherichia coli.

\section{References}

1. Stevens MP, Frankel GM. The locus of enterocyte effacement and associated virulence factors of enterohemorrhagic Escherichia coli. Microbiol Spectr. 2014;2:EHEC-0007-2013.

2. Frank C, Werber D, Cramer JP, Askar M, Faber M, an der Heiden M, et al. HUS Investigation Team. Epidemic profile of Shiga-toxin-producing Escherichia coli O104:H4 outbreak in Germany. N Engl J Med. 2011;365:1771-80. https://doi.org/10.1056/NEJMoa1106483

3. Paton AW, Woodrow MC, Doyle RM, Lanser JA, Paton JC. Molecular characterization of a Shiga toxigenic Escherichia coli O113:H21 strain lacking eae responsible for a cluster of cases of hemolytic-uremic syndrome. J Clin Microbiol. 1999;37:3357-61. https:// doi.org/10.1128/JCM.37.10.33573361.1999

4. Schifferli A, von Vigier RO, Fontana M, Spartà G, Schmid H, Bianchetti MG, et al.; Swiss Pediatric Surveillance Unit. Hemolytic-uremic syndrome in Switzerland: a nationwide surveillance 1997-2003. Eur J Pediatr. 2010;169:591-8. https://doi.org/10.1007/s00431-009-1079-9

5. Iguchi A, Nishii H, Seto K, Mitobe J, Lee K, Konishi N, et al. Additional Og-typing PCR techniques targeting E. coli-novel and Shigella-unique $\mathrm{O}$-antigen biosynthesis gene clusters. J Clin Microbiol. 2020;58:e01493-20. https:/ / doi.org/ 10.1128/JCM.01493-20

6. DebRoy C, Fratamico PM, Yan X, Baranzoni G, Liu Y, Needleman DS, et al. Comparison of O-antigen gene clusters of all O-serogroups of Escherichia coli and proposal for adopting a new nomenclature for O-typing. PLoS One. 2016;11:e0147434-e.

7. Lee K, Izumiya H, Iyoda S, Ohnishi M. Effective surveillance using multilocus variable-number tandem-repeat analysis and whole-genome sequencing for enterohemorrhagic Escherichia coli O157. Appl Environ Microbiol. 2019;85:e00728-19. https:// doi.org/10.1128/AEM.00728-19

8. Kimata K, Lee K, Watahiki M, Isobe J, Ohnishi M, Iyoda S. Global distribution of epidemic-related Shiga toxin 2 encoding phages among enteroaggregative Escherichia coli. Sci Rep. 2020;10:11738. https://doi.org/10.1038/s41598-020-68462-9

9. Yoshimura D, Kajitani R, Gotoh Y, Katahira K, Okuno M, Ogura Y, et al. Evaluation of SNP calling methods for closely related bacterial isolates and a novel high-accuracy pipeline: BactSNP. Microb Genom. 2019;5:e000261. https:/ / doi.org/10.1099/mgen.0.000261

10. Croucher NJ, Page AJ, Connor TR, Delaney AJ, Keane JA, Bentley SD, et al. Rapid phylogenetic analysis of large samples of recombinant bacterial whole genome sequences using Gubbins. Nucleic Acids Res. 2015;43:e15. https://doi.org/10.1093/nar/gku1196

11. Gonzalez-Escalona N, McFarland MA, Rump LV, Payne J, Andrzejewski D, Brown EW, et al. Draft genome sequences of two O104:H21 Escherichia coli isolates causing hemorrhagic colitis during a 1994 Montana outbreak provide insight into their pathogenicity. Genome Announc. 2013;1:e00805-13. https:/ / doi.org/10.1128/genomeA.00805-13

12. Banjo M, Iguchi A, Seto K, Kikuchi T, Harada T, Scheutz F, et al.; Pathogenic E. coli Working Group in Japan. Escherichia coli H-genotyping PCR: a complete and practical platform for molecular H typing. J Clin Microbiol. 2018;56:e00190-18. https:// doi.org/10.1128/JCM.00190-18

13. Iguchi A, Iyoda S, Seto K, Nishii H, Ohnishi M, Mekata H, et al. Six novel $O$ genotypes from Shiga toxin-producing Escherichia coli. Front Microbiol. 2016;7:765. https://doi.org/ 10.3389/fmicb.2016.00765

14. Lee K, Morita-Ishihara T, Iyoda S, Ogura Y, Hayashi T, Sekizuka T, et al.; EHEC Working Group in Japan. A geographically widespread outbreak investigation and development of a rapid screening method using whole genome sequences of enterohemorrhagic Escherichia coli O121. Front Microbiol. 2017;8:701. https://doi.org/10.3389/fmicb.2017.00701

15. Holmes A, Allison L, Ward M, Dallman TJ, Clark R, Fawkes A, et al. Utility of whole-genome sequencing of Escherichia coli $\mathrm{O} 157$ for outbreak detection and epidemiological surveillance. J Clin Microbiol. 2015;53:356573. https://doi.org/10.1128/JCM.01066-15

Address for correspondence: Kenichi Lee, Department of

Bacteriology I, National Institute of Infectious Diseases, 1-23-1,

Toyama, Shinjuku, Tokyo, 1628640, Japan; email: leek@niid.go.jp 